\title{
Riemann-Problem and Level-Set Approaches for Homentropic Two-Fluid Flow Computations
}

\author{
B. Koren,* M. R. Lewis, ${ }^{* 1}$ E. H. van Brummelen, ${ }^{* 2}$ and B. van Leer ${ }^{+1}$ \\ * (WL PO B Box 94()79), I()90 GB Amsterdam, The Netherlands; and TUniversity of Michigan, Department \\ of Aerospace Engineering, Ann Arbor, Michigan 48109-2140 \\ E-mail: barry.koren@cwi.nl, mervyn.lewis@cwi.nl, e.h.vanbrummelen@lr.tudelft.nl. \\ and bram@engin.umich.edu
}

Received September 4, 2001 ; revised April 1, 2002

\begin{abstract}
A tinite-volume method is presented for the computation of compressible flows of two immiscible fluids at very different densities. A novel ingredient in the method is a linearized, two-lluid Osher scheme, allowing for flux computations in the case of different fluids (e.g., water and air) left and right of a cell face. A level-set technique is employed to distinguish between the two fluids. The level-set equation is incorporated into the system of hyperbolic conservation laws. Fixes are presented for the solution crrors (pressure oscillations) that may occur near two-fluid interfaces when applying a capturing method. The fixes are analyzed and tested. For two-fluid flows with arbitrarily large density ratios, a simple variant of the ghost-fluid method appears to he a perfect remedy. Computations for compressible water-air flows yield perfectly sharp, pressure-oscillation-free interfaces. The masses of the separate fluids appear to be conserved up to first-order accuracy. (c) 2002 Elsevier Science iUSA)

Key Words: free surfaces, compressible liquid-gas flows, interface capturing, Osher scheme, level-set method, interface-pressure error, ghost-fluid method.
\end{abstract}

\section{INTRODUCTION}

The present paper is directed towards an efficient, physically correct finite-volume computation of the flow of two compressible fluids, e.g., water and air, at uniformly subsonic speeds. Other premises are that the two fluids do not mix, that vaporization and condensation phenomena do not occur and that surface tension can be neglected.

In recent years, various papers have been published that present specific finite-volume methods for two-fluid flow computations. In most of these papers, a two-fluid flux formula is

1 Supported by the Dutch Technology Foundation STW.

Supported by the Maritime Research Institute Netherlands. Current address: Delft University of Technology, Faculty of Aerospace Engineering, P.O. Box 5058, 2600 GB Delft, The Netherlands. 
proposed in which an approximate Riemann solver is applied. In [1. 10]. Roe-type schemes are proposed and in [14], Rusanov-type and Harten-Lax-van-Leer-type schemes. In the present paper, an Osher-type two-fluid flux formula is proposed. The formula is extremels simple and computationally very efficient. At the boundaries of the computational domain. it is completely consistent with that in the interior. The flux formula incorporates a level-set term for accurately capturing the two-fluid interface.

A known difficulty of capturing contact discontinuities in a conservative formulation of the compressible, two-fluid Euler equations is that large solution errors coften called pressure oscillations) may arise near the contact discontinuity. For incompressible two-fluid flow computations (see, e.g., [17]) the pressure-oscillation problem is much les severe than for compressible since density is not used for further computations, such as the computation of pressure from an equation of state. For tracking and fitting approacher the pressure-oscillation problem does not exist at all. As far as conservative capturing method. for compressible two-fluid flows are concerned, we remark that not all of these methud are necessarily cursed with the pressure-oscillation problem: in [3] we propose a fully conservative formulation which, without any measure being taken, has no pressure error at two-fluid interfaces.

Without remedial intervening, the conservative formulation considered in the present paper also suffers from the pressure-oscillation problem. We will show this on the basis of a model flow with a known exact solution. The solution error is proportional to the densit! ratio across the interface. For large density jumps across the interface, the error may even degenerate to instability. Fixes for the solution-error problem can be found in the literature We refer to the works of Karni $[10,11]$ and Abgrall [1], their common paper [21, and 15.4. 141. In most of the available literature though, the ratio of the two densities at the interfice is $\mathcal{O}(1)-\mathcal{O}\left(1()^{2}\right)$. To our knowledge, only in $[5,14]$ ratios of $\mathcal{O}\left(10^{3}\right)$, typical water-air ratios. are considered. In the current paper a simple fix is proposed, which allows arbitrarily large density ratios.

The contents of the paper is the following. In Section 2, the continuous flow model is given: conservation laws, equations of state (for water and air). and level-set equation. In Section 3, the space discretization of the equations is presented (the Riemann problem and the corresponding Godunov-type scheme, at both interior and boundary cell faces. Nev. in Section 4, we analyze the solution-error problem near interfaces. In Section 5 , some approaches to fix the problem are addressed. Not all of these approaches isome already known appear to work for water-air flow with its large density jump. One tix is proposed which works perfectly, it is a simple variant of the ghost-fluid method [5]. In Section 6. numerical results are presented for compressible water-air flows. In Section 7. we outline the extension of our method to multidimensional problems, and in Section 8 the paper is concluded.

\section{FLOW MODEL}

\subsection{Conservation Equations}

In $1 D$, for a sufficiently small control volume $\Omega$, conservation of mass and momentum reads

$$
\int_{\Omega} \frac{\partial}{\partial t}\left(\begin{array}{c}
\rho \\
\rho u
\end{array}\right) d x+\left(\begin{array}{c}
\rho u \\
\rho u^{2}+p
\end{array}\right)_{i \Omega_{\mathrm{n} q u}}-\left(\begin{array}{c}
\rho u \\
\rho u^{2}+p
\end{array}\right)_{i \Omega_{w} u}=0
$$


with $\rho$ the bulk density

$$
\rho=\alpha(x, t) \rho_{w}(p)+(1-\alpha(x, t)) \rho_{a}(p),
$$

where $\alpha$ is, e.g., the volume-of-water fraction and $\rho_{u}(p)$ and $\rho_{l}(p)$ are the equations of state for water and air, respectively. To balance system (1) and (2), the equations of state and an equation determining the location of the interface (and hence $\alpha$ ) still have to be chosen.

With the above bulk-density formulation, in a finite-volume discretization, total mass of the fluid will be conserved, but not necessarily the masses of the two separate fluids. When $\alpha(x, t)$ is poorly resolved, the two separate masses are poorly conserved as well. Hence, an accurate resolution of the interface location(s) is of paramount importance. For this purpose, we follow a level-set approach, to be discussed in the next section.

\subsection{Level-Set Equation}

To accurately resolve the interface location(s), a level-set approach [16] is more appropriate than the classical volume-of-fluid (VOF) approach [8] because of its better smoothness (and thus accuracy) properties at precisely the point of interest: the interface. Good smoothness of the level-set function is first taken care of in the level-set function's initialization. A common approach is to initialize the level-set function as the signed distance to the initial interface, with the distance positive in, e.g., water and negative in air. (To get uniformly smooth level-set functions in the case of multiple interfaces, advantage may be taken of nonlinear initializations [12].)

Keeping the level-set function smooth requires some attention. During the computation, the level-set function may need to be regularized. In this reinitialization step, care needs to be taken that the free-surface location is preserved. In brief, the reinitialization can be done as follows. After one or more time steps the locations of the interfaces are determined as the zeros of the level-set function. Next, the level-set function is simply reinitialized as the signed distance to the nearest interface.

Denoting the level-set function by $\phi$, in 1D, it is advected by

$$
\frac{\partial \phi}{\partial t}+u \frac{\partial \phi}{\partial x}=0 .
$$

Combined with the bulk-mass conservation equation from (1), advection equation (3) may be written in the conservative control-volume form

$$
\int_{\Omega} \frac{\partial(\rho \phi)}{\partial t} d x+(\rho u \phi)_{\partial \Omega_{\mathrm{regh}}}-(\rho u \phi)_{i \mathrm{i} \Omega_{\mathrm{leth}}}=0 .
$$

Conservation of $\rho \phi$ is not physical; there is no conservation law for it. The form (4) is simply practical because it is consistent with system (1); it can be directly embedded into it. With $\phi(x, t)$ known, the VOF function $\alpha$ can be computed for any finite volume. In Section 3.2, for an equidistant ID finite-volume grid, $\alpha=\alpha(\phi)$ is worked out in detail; in Section 7.1 we outline the extension to multiple dimensions (multi-D).

Summarizing, the VOF fraction is used, not the VOF method. In the VOF method, a transport equation for $\alpha$ is used. Instead, here we apply a transport equation for $\phi$ (in the consistent form of a conservation equation for $\rho \phi$ ). 


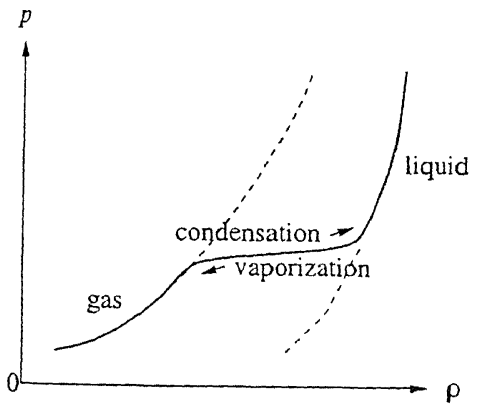

a. For two-phase flows

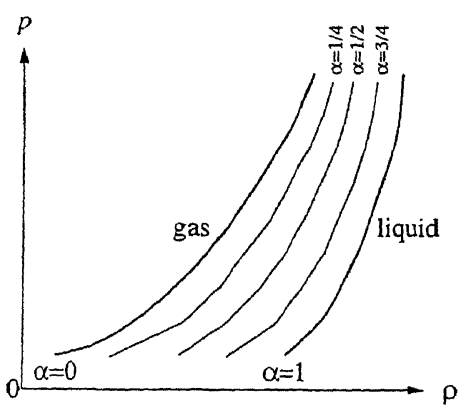

b. For immiscible twa-fluid flows

FIG. 1. Pressure-density diagrams.

\subsection{Equation of State}

In homentropic water-air computations, for both fluids, elegant use can be made of a single equation of state, viz. Tait's,

$$
\frac{p+B p_{\mathrm{ref}}}{(1+B) p_{\mathrm{ref}}}=\left(\frac{\rho}{\rho_{\mathrm{ref}}}\right)^{\gamma} \text {, }
$$

where the subscript ref indicates some reference state. The reference pressure $p_{\text {ref }}$ is chosen freely but equally for the two fluids. The value of $\rho_{\text {ref }}$ for each of the two fluids corresponds with $p_{\text {ref }}$ and is read from standard data bases for fluid properties. Concerning the material constants $\gamma$ and $B$, for water: $\gamma=7$ and $B=3000$, and for air $\gamma=\frac{7}{5}$ and $B=0$. With (5), both the water and air densities, $\rho_{u^{\prime}}(p)$ and $\rho_{a}(p)$, are convex functions of pressure. So is the corresponding bulk density $\rho$ according to (2). The physical consequences of this overall convexity are that neither locally low speeds of sound (lower than in pure water or pure air) nor expansion shocks can occur. In two-phase approaches these phenomena can occur [7]. For these flows, the pressure-density diagram may look as sketched in Fig. la, i.e., as a mixed convex-concave curve with extremely small values of the speed of sound, $c=d p / d \rho$, in the condensation/vaporization zone. As opposed to that, in the present case of two immiscible fluids, a family of purely convex curves exists, curves that become increasingly steep for increasing $\alpha$ (Fig. 1b). So, for any $p$ and for all values of $\alpha \in(0,1)$ it holds that $c_{a}<c<c_{u}$. A slight inconvenience of using (2) in combination with (5) is that the calculation of $p$ for known $\rho$ and $\alpha(\alpha \neq 0$ and $\alpha \neq 1)$ needs to be done iteratively.

\section{DISCRETIZATION}

\subsection{Finite Volumes}

Summarizing, for a (sufficiently small) control volume $\Omega$, the system of equations considered reads

$$
\int_{\Omega} \frac{\partial q}{\partial t} d x+(f(q))_{i \Omega_{\mathrm{n} ! \mathrm{ht}}}-(f(q))_{i \Omega_{\mathrm{lcth}}}=0, \quad q=\left(\begin{array}{c}
\rho \\
\rho u \\
\rho \phi
\end{array}\right), \quad f(q)=\left(\begin{array}{c}
\rho u \\
\rho u^{2}+p \\
\rho u \phi
\end{array}\right),
$$




$$
\begin{aligned}
\rho & =\alpha(\phi) \rho_{w}(p)+(1-\alpha(\phi)) \rho_{a}(p), \\
\rho_{w^{\prime}}(p) & =\left(\frac{p+B_{w} p_{\mathrm{ref}}}{\left(1+B_{w}\right) p_{\mathrm{ref}}}\right)^{\frac{1}{\gamma^{\prime \prime}}}\left(\rho_{w}\right)_{\mathrm{ref}}, \quad \rho_{u}(p)=\left(\frac{p}{p_{\mathrm{ref}}}\right)^{\frac{1}{v^{w}}}\left(\rho_{u}\right)_{\mathrm{ref}} .
\end{aligned}
$$

with $\alpha(\phi)$ the fraction of the size of $\Omega$ over which $\phi \geq 0$. Two early papers that considered the computation of compressible water-air flows are $[4,6]$. A basic difference between the present two-fluid flow equations and those from $[4,6]$ is that in the latter the third equation is the energy equation, whereas here it is the level-set equation.

\subsection{Velume-of-Fluid Fraction}

The natural space discretization for (6) is a finite-volume technique. For convenience, we consider cell-centered finite volumes with constant mesh size. This choice directly allows us to work out the discretization of $\alpha(\phi)$. Consider finite volume $\Omega_{i}$ and its left and right neighbors, $\Omega_{i-1}$ and $\Omega_{i+1}$, respectively, and define the level-set values at the cell faces $\partial \Omega_{i-\frac{1}{3}}$ and $\partial \Omega_{i+\frac{1}{3}}$ as

$$
\phi_{i-\frac{1}{2}}=\frac{1}{2}\left(\phi_{i-1}+\phi_{i}\right), \quad \phi_{i+\frac{1}{2}}=\frac{1}{2}\left(\phi_{i}+\phi_{i+1}\right) .
$$

Then, for example, for $\phi_{i} \geq 0$, we propose the four $\alpha_{i}$ possibilities given in Fig. 2.

So, in determining $\phi_{i-\frac{1}{2}}$ and $\phi_{i+\frac{1}{2}}$, as well as $x(\phi=0)$, we make use of piecewise linear interpolation of $\phi$. The linear interpolation is exact as long as the level-set function is the signed-distance function.

\subsection{Riemann-Problem Approach}

The challenge of a finite-volume formulation is to choose or devise a physically correct (wo-fluid flux formula to apply at the low discrete level of cell faces. The exact solution of the 1D Riemann problem at each cell face, the well-known Godunov approach, requires the use of a numerical root finder. We avoid this by considering an approximate Riemann solver. For this, we prefer a two-fluid version of Osher's [13], particularly because of its consistent boundary-condition treatment. Denoting the left and right cell-face states by $q_{0}$
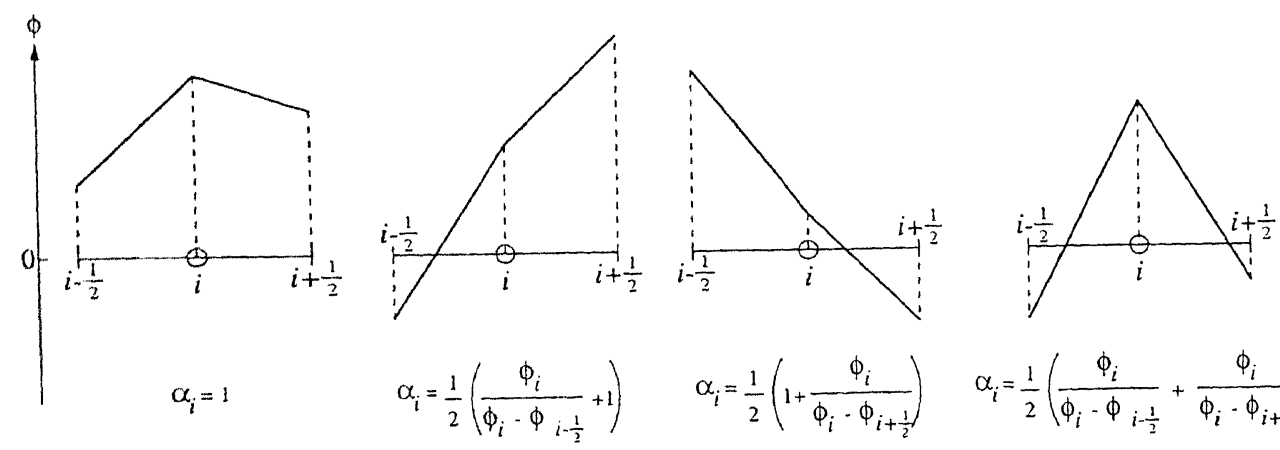

$$
\alpha_{i}=\frac{1}{2}\left(1+\frac{\phi_{i}}{\phi_{i} \cdot \phi_{i+\frac{1}{2}}}\right)
$$

$$
\alpha_{i}=\frac{1}{2}\left(\frac{\phi_{i}}{\phi_{i}-\phi_{i-\frac{1}{2}}}+\frac{\phi_{i}}{\phi_{i}-\phi_{i+\frac{1}{2}}}\right)
$$

FIC: 2. Four possible combinations of signs of $\phi_{t}-\frac{1}{2}$ and $\phi_{i}, \frac{1}{2}$, for $\phi_{i} \geq 0$, together with corresponding formulate lior VOF fraction $\alpha_{1}$. 
and $q_{1}$ and the flux formula by $F\left(q_{0}, q_{1}\right)$, the Osher scheme may be written as

$$
F\left(q_{0}, q_{1}\right)=f\left(q_{0}\right)+\int_{q_{0}}^{q_{1}} \frac{d f^{-}}{d q} d q
$$

with $d f^{-} / d q$ the negative eigenvalue part of $d f / d q$. The eigenvalues of the present Jacobian are $\lambda_{1}=u-\sqrt{\partial p / \partial \rho}, \lambda_{2}=u$, and $\lambda_{3}=u+\sqrt{\partial p / \partial \rho}$. $(\partial p / \partial \phi$ does not occur in the wave speeds.) The Riemann-invariant relations describing the two intermediate states $q_{\frac{1}{3}}$ and $q_{\frac{2}{3}}$ along the wave path in state space for $\lambda_{1}, \lambda_{2}$, and $\lambda_{3}$ successively are

$$
\begin{gathered}
u_{\frac{1}{3}}+\int^{\rho_{\frac{1}{3}}} \frac{1}{\rho} \sqrt{\frac{\partial p}{\partial \rho}} d \rho=u_{0}+\int^{p_{1}} \frac{1}{\rho} \sqrt{\frac{\partial p}{\partial \rho}} d \rho, \quad \phi_{\frac{1}{3}}=\phi_{0}, \\
u_{\frac{1}{3}}=u_{\frac{2}{3}}=u_{\frac{1}{2}}, \quad p_{\frac{1}{3}}=p_{\frac{2}{3}}=p_{\frac{1}{2}}, \\
u_{\frac{2}{3}}-\int^{\rho_{\frac{2}{3}}} \frac{1}{\rho} \sqrt{\frac{\partial p}{\partial \rho}} d \rho=u_{1}-\int^{\rho_{1}} \frac{1}{\rho} \sqrt{\frac{\partial p}{\partial \rho}} d \rho, \quad \phi_{\frac{2}{3}}=\phi_{1} .
\end{gathered}
$$

Hence, the level-set function $\phi$ can only change along the subpath corresponding to the eigenvalue $\lambda_{2}$, i.e., across the contact discontinuity. It is invariant along the outer subpaths; physically speaking, if $\phi$ is the signed-distance function, along the outer subpaths the distance to the two-fluid interface is constant. The integrals in (9a) and (9c) can be written explicitly for the equations of state given in (6c). However, when, e.g., a water-air interface is captured, the explicit calculation of $u_{\frac{1}{2}}$ and $p_{\frac{1}{2}}$ is hampered by nonlinearity; a transcendental equation then needs to be solved.

\subsection{Linearized, Two-Fluid Osher Scheme}

Since $\phi$ is constant along the two outer subpaths of the wave path, along both subpaths the bulk density can only vary due to pressure changes. For flows that are low-subsonic, large pressure and hence large bulk-density changes will not occur and, consequently, the integrals

$$
\int^{\rho_{\frac{1}{3}}} \frac{1}{\rho} \sqrt{\frac{\partial p}{\partial \rho}} d \rho \quad \text { and } \quad \int^{\rho \frac{2}{3}} \frac{1}{\rho} \sqrt{\frac{\partial p}{\partial \rho}} d \rho
$$

can be linearized by good approximation around $\rho_{0}$ and $\rho_{l}$, respectively, yielding for $(9 a)-$ (9c)

$$
u_{\frac{1}{2}}=u_{0}-\left(\rho_{\frac{1}{3}}-\rho_{0}\right) \frac{c_{0}}{\rho_{0}}, \quad u_{\frac{1}{2}}=u_{1}+\left(\rho_{\frac{2}{3}}-\rho_{1}\right) \frac{c_{1}}{\rho_{1}} .
$$

Likewise, $p_{\frac{1}{2}}$ can be linearized around $\rho_{0}$ and $\rho_{1}$ as

$$
p_{\frac{1}{2}}=p_{0}+\left(\rho_{\frac{1}{3}}-\rho_{0}\right) c_{0}^{2}, \quad p_{\frac{1}{2}}=p_{1}+\left(\rho_{\frac{2}{3}}-\rho_{1}\right) c_{1}^{2} .
$$
Elimination of $\rho_{\frac{1}{3}}-\rho_{0}$ and $\rho_{\frac{2}{3}}-\rho_{1}$ from (10) and (11) gives $\frac{p_{\frac{1}{2}}-p_{0}}{u_{\frac{1}{2}}-u_{0}}=-\rho_{0} c_{0}$ and $\frac{p_{\frac{1}{2}}-p_{1}}{u_{\frac{1}{2}}-u_{1}}=$
$\rho_{1} c_{1}$, i.e.,

$$
\left(\begin{array}{c}
u \frac{1}{2} \\
p_{\frac{1}{2}}
\end{array}\right)=\left(\begin{array}{c}
\frac{c_{0} u_{0}+c_{1} u_{1}+\left(p_{0}-p_{1}\right)}{c_{0}+C_{1}} \\
\frac{c_{1} p_{0}+C_{0} p_{1}+C_{0} c_{1}\left(u_{0}-u_{1}\right)}{C_{0}+C_{1}}
\end{array}\right), \quad C_{0} \equiv \rho_{0} c_{0}, \quad C_{1} \equiv \rho_{1} c_{1}
$$


For the density and level-set function in the two intermediate points it holds that

$$
\left(\begin{array}{c}
\rho_{\frac{1}{3}} \\
\phi_{\frac{1}{3}}
\end{array}\right)=\left(\begin{array}{c}
\rho_{0}+\frac{p_{\frac{1}{2}}-p_{0}}{c_{0}^{2}} \\
\phi_{0}
\end{array}\right),\left(\begin{array}{c}
\rho_{\frac{2}{3}} \\
\phi_{\frac{2}{3}}
\end{array}\right)=\left(\begin{array}{c}
\rho_{1}+\frac{p_{\frac{1}{2}}-p_{1}}{c_{1}^{2}} \\
\phi_{1}
\end{array}\right) .
$$

Ignoring all supersonic possibilities among all possible combinations of signs of $u_{0}-c_{()}$, $u_{\frac{1}{2}}-c_{\frac{1}{3}}, u_{\frac{1}{2}}+c_{\frac{2}{3}}$, and $u_{1}+c_{1}$ (note the consequent efficiency improvement in checking eigenvalue signs), the linearized, two-fluid scheme reads

$$
F\left(q_{0}, q_{1}\right)_{u_{\frac{1}{2}} \geq 0}=\left(\begin{array}{c}
\rho_{\frac{1}{3}} u_{\frac{1}{2}} \\
\rho_{\frac{1}{3}} u_{\frac{1}{2}}^{2}+p_{\frac{1}{2}}^{2} \\
\rho_{\frac{1}{3}} u_{\frac{1}{2}} \phi_{\frac{1}{3}}
\end{array}\right), \quad F\left(q_{0}, q_{1}\right)_{\left.u_{\frac{1}{2}} \leq 0\right)}=\left(\begin{array}{c}
\rho_{\frac{2}{3}} u_{\frac{1}{2}} \\
\rho_{\frac{2}{3}} u_{\frac{1}{2}}^{2}+p_{\frac{1}{2}} \\
\rho_{\frac{2}{3}} u_{\frac{1}{2}} \phi_{\frac{2}{3}}
\end{array}\right) .
$$

We remark that the real nonlinear flux functions $f\left(q_{\frac{1}{3}}\right)$ and $f\left(q_{\frac{2}{3}}\right)$ are applied and not

$$
\begin{aligned}
& F\left(q_{0}, q_{1}\right)_{u_{\frac{1}{2}} \geq 0}=f\left(q_{0}\right)+\left(q_{\frac{1}{3}}-q_{0}\right) \frac{d f\left(q_{0}\right)}{d q}, \\
& F\left(q_{0}, q_{1}\right)_{u_{\frac{1}{2}} \leq 0}=f\left(q_{1}\right)+\left(q_{\frac{2}{3}}-q_{1}\right) \frac{d f\left(q_{1}\right)}{d q} .
\end{aligned}
$$

There is no need for the latter linearized formulae. On the contrary, as opposed to (12)-(14), they may give rise to an erroneous, ambiguous flux at $u_{\frac{1}{2}}=0$ (steady contact discontinuity); $f\left(q_{0}\right)+\left(q_{\frac{1}{3}}-q_{0}\right)\left[d f\left(q_{0}\right) / d q_{1}\right]$ and $f\left(q_{1}\right)+\left(q_{\frac{2}{3}}-q_{1}\right)\left[d f\left(q_{1}\right) / d q_{1}\right]$ may differ for $u_{\frac{1}{2}}=0$.

\subsection{Boundary-Condition Treatment}

A very favorable property of the Osher scheme is that the fluxes across the boundary faces can be computed with the same formula as that for the interior faces, i.e., with (14). Denoting the state at the boundary by $q_{b}$, in the case of a left boundary $q_{0}=q_{b}$, and in the case of a right $q_{1}=q_{b}$. We work out the inflow and outflow boundaries and the nonpermeable boundary as a limit case. For all three it holds that for boundaries at the left and right, respectively,

$$
\frac{p_{b}-p_{1}}{u_{b}-u_{1}}=C_{1}, \quad \frac{p_{b}-p_{()}}{u_{b}-u_{0}}=-C_{0} .
$$

3.5.1. Inflow boundary. From (15), it follows that the two boundary conditions to be imposed here cannot be $u_{b}$ and $p_{b}$ simultaneously; when $u_{b}$ is imposed, $p_{h}$ follows, and vice versa. Hence, the second boundary condition must be one for $\phi_{b}$. To compute the boundary flux, the OD bulk density $\rho_{b}$ still needs to be defined. An appropriate OD choice is

$$
\left(\rho_{b}\right)_{\phi_{b} \geq 0}=\rho_{u \prime}\left(p_{b}\right), \quad\left(\rho_{b}\right)_{\phi_{b}<0}=\rho_{a}\left(p_{b}\right) .
$$

3.5.2. Outflow boundary: Here, in addition to (15), the equations

$$
\phi_{b}=\phi_{0}, \quad \phi_{b}=\phi_{1}
$$

are available. So, the single boundary condition to be imposed must be $u_{b}$ or $p_{b}$, or some combination of both. The bulk density $\rho_{b}$ is defined as in the inflow case. 
3.5.3. Nonpermeable boundary: At a nonpermeable boundary (at least) $u_{b}=0$ must be imposed, which, given (15), already determines $p_{h}$. Considering a nonpermeable boundary as the limit case of an inflow boundary, $\phi_{b}$ must still be imposed. Considering it as the limit of outflow, $\phi_{b}$ follows from the interior solution $\left(\phi_{b}=\phi_{1}\right.$ for a left boundary and $\phi_{b}=\phi_{0}$ for a right). The outflow-limit case is to be preferred. As opposed to the inflow-limit case, it allows the interface to freely move along the nonpermeable boundary. Also here, the bulk density may be defined according to (16).

\section{ERROR NEAR INTERFACE}

In the present section we analyze the pressure-oscillation problem for the equations in system (6). Similar analyses for other systems of equations have already been given in [2, $9,10]$.

\subsection{Analysis for Model Flow.}

Consider a ID tube with unit length, $x \in[0,1]$, inflow of water at $x=0$, outflow at $x=1$, and the initial solution

$$
\begin{aligned}
& u(x, t=0)=U>0, \\
& \rho(x, t=0)= \begin{cases}\rho_{u}(P), & p(x, t=0)=P, \\
\rho_{a}(P) . & x>\left(x_{\mathrm{fs}}\right)_{t=0},\end{cases}
\end{aligned}
$$

where $U$ and $P$ are constant and where $x_{\mathrm{fs}}$ is the location of the free surface, i.e., the waterair interface. For $t>0$, the corresponding exact Euler flow solution reads $u(x, t)=U$, $p(x, t)=P, \rho(x, t)=\rho_{u}(P)$ for $x \leq\left(x_{\mathrm{fs}}\right)_{t=0}+U t$ and $\rho(x, t)=\rho_{a}(P)$ for $x>\left(x_{\mathrm{fs}}\right)_{t=0}+$ $U t$. This simple model flow precisely uncovers the deficiency of capturing methods with regard to material interfaces. For the space discretization of (6) we consider an equidistant finite-volume grid with mesh size $h$. For the time integration, the forward-Euler scheme is taken. The space discretization is taken as first-order accurate. Then, denoting the solution in cell $i$ at the old time level by $q_{i}^{\prime \prime}, q=(\rho, \rho u, \rho \phi)^{T}$, the equation for the solution $q_{i}^{n+1}$ at the new time level is

$$
q_{i}^{n+1}=q_{i}^{n}-\frac{\Delta t}{h}\left(F\left(q_{i}^{\prime \prime}, q_{i+1}^{n}\right)-F\left(q_{i-1}^{n}, q_{i}^{n}\right)\right)
$$

with $\Delta t$ the time step and $F$ the linearized, two-fluid Osher flux (14). Considering the situation where $q_{i-1}^{\prime \prime}, q_{i}^{n}$, and $q_{i+1}^{\prime \prime}$ are according to the initial solution (18), with $\left(x_{\mathrm{fs}}\right)^{n}=$ $x_{i-\frac{1}{2}}$ (Fig. 3), (19) yields

$$
\left(\begin{array}{c}
\rho \\
\rho u \\
\rho \phi
\end{array}\right)_{i}^{n+1}=\sigma \rho_{u}(P)\left(\begin{array}{c}
1 \\
U \\
\frac{h}{2}
\end{array}\right)+(1-\sigma) \rho_{u}(P)\left(\begin{array}{c}
1 \\
U \\
-\frac{h}{2}
\end{array}\right), \quad \sigma \equiv \frac{U \Delta t}{h}
$$

Note that $\rho_{i}^{n+1}$ according to (20) is exact. as is $u_{i}^{n+1}=(\rho u)_{i}^{n+1} / \rho_{i}^{n+1}=U$. However, for 


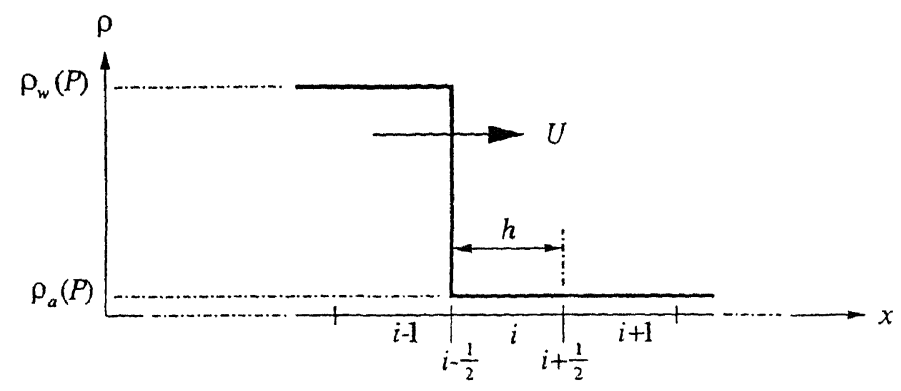

FIG. 3. Bulk-density distribution near cell $i$ at time level $n$ and water-air interface at $x+\frac{1}{2}$.

$\phi_{i}^{n+1}$ it follows from (20) that

$$
\phi_{i}^{n+1}=\frac{(\rho \phi)_{i}^{n+1}}{\rho_{i}^{n+1}}=\frac{\sigma \rho_{w}-(1-\sigma) \rho_{a}}{\sigma \rho_{w}+(1-\sigma) \rho_{a l}} \frac{h}{2}
$$

whereas the exact discrete solution reads $\left(\phi_{i}^{n+1}\right)_{\text {exact }}=-h / 2+\sigma h$. Hence, for the local discretization error $\Delta \phi_{i}^{n+1}=\phi_{i}^{n+1}-\left(\phi_{i}^{n+1}\right)_{\text {exact }}$ it holds that

$$
\Delta \phi_{i}^{n+1}=\sigma(1-\sigma) \frac{\rho_{u}-\rho_{u}}{\sigma \rho_{w^{\prime}}+(1-\sigma) \rho_{u}} h .
$$

So, $\Delta \phi_{i}^{n+1}=0$ only if $\sigma=0$ (trivial), $\sigma=1$, or $\rho_{u^{\prime}}=\rho_{u}$. The local discretization error (22) is $\mathcal{O}(h)$, but cannot be made of higher order by applying a higher order discretization. (This holds for any numerical flux function $F$.) Higher than first-order accuracy is simply inhibited by the bulk density, which is a smeared representation of the exact discrete density. Through bulk-density formula (6b), the error (22) carries over into a pressure error $\Delta p_{i}^{n+1}$. Given $\Delta \rho_{i}^{n+1}=0$, from (6b) it follows after linearization that

$$
\Delta p_{i}^{n+1}=\frac{-c_{u}^{2} c_{a}^{2}}{\left(\alpha_{i}^{n+1}+\Delta \alpha_{i}^{n+1}\right) c_{a}^{2}+\left(1-\alpha_{i}^{n+1}-\Delta \alpha_{i}^{n+1}\right) c_{w 1}^{2}}\left(\rho_{w}-\rho_{u}\right) \Delta \alpha_{i}^{n+1}
$$

From Fig. 2 we know that besides depending on $\Delta \phi_{i}^{n+1}, \Delta \alpha_{i}^{n+1}$ also depends on $\Delta \phi_{i-1}^{n+1}$ and $\Delta \phi_{i+1}^{n+1}$. For the model flow considered, it follows with the current scheme that $\phi_{i-1}^{n+1}=$ $h / 2+\sigma h$ and $\phi_{i+1}^{n+1}=-3 h / 2+\sigma h$, which are both the exact results. With the formulae from Fig. 2, the expressions found for the error $\Delta \alpha_{i}^{n+1}=\alpha_{i}^{n+1}-\left(\alpha_{i}^{n+1}\right)_{\text {exacl }}$ are

$$
\begin{aligned}
& \left(\Delta \alpha_{i}^{n+1}\right)_{\phi_{i}^{n+1} \geq 0}=\left(\frac{3}{2}-\sigma\right) \frac{\Delta \phi_{i}^{n+1}}{h+\Delta \phi_{i}^{n+1}}, \\
& \left(\Delta \alpha_{i}^{n+1}\right)_{\phi_{i}^{n+1}<0}=\left(\frac{1}{2}+\sigma\right) \frac{\Delta \phi_{i}^{n+1}}{h-\Delta \phi_{i}^{n+1}} .
\end{aligned}
$$

So, $\Delta \alpha_{i}^{n+1}=\mathcal{O}\left(h^{0}\right)$, say $\mathcal{O}(1)$, and hence with (23), the pressure error $\Delta p_{i}^{n+1}$ also is $\mathcal{O}(1)$. Moreover, the pressure error (23) is proportional to the density ratio $\rho_{w} / \rho_{a}$. 


\subsection{Guidelines for Error Improvements}

Before proposing ways to improve the poor local error behavior near the interface, it is useful to consider the conservative equations near the two-fluid interface and to make an error analysis of bulk-density relation (6b).

4.2.1. Fluid-flow equations near interface. Consider the situation in which the interface is in cell $\Omega=\Omega_{i}$ (only the interface, so no shock or rarefaction). Since velocity and pressure are continuous across the interface, for sufficiently small $\Omega_{i}$, we may then write by good approximation $u_{i-\frac{1}{2}}=u_{i+\frac{1}{2}}=u_{i}$ and $p_{i-\frac{1}{2}}=p_{i+\frac{1}{2}}$. With this, (1) can be rewritten as a system of advection equations for the entire solution vector $q_{i}$,

$$
\int_{\Omega_{1}} \frac{\partial q}{\partial t} d x+u_{i}\left(q_{i+\frac{1}{2}}-q_{i-\frac{1}{2}}\right)=0 .
$$

Contact discontinuities are linear phenomena. If all conservative solution components are advected, $\rho_{i},(\rho u)_{i}$, and $(\rho \phi)_{i}$, then any solution component in $\Omega_{i}$ (either conservative or nonconservative) is advected. That is, in (25) for a cell with only a contact discontinuity, instead of the fully conservative solution representation $q_{i}=\left(\rho_{i},(\rho u)_{i},(\rho \phi)_{i}\right)$ we may equally well consider, e.g., the partially conservative representation $q_{i}=\left(\rho_{i},(\rho u)_{i}, \phi_{i}\right)$, the fully nonconservative representation $q_{i}=\left(u_{i}, p_{i}, \phi_{i}\right)$, or other representations.

4.2.2. Error analysis of bulk-density relation. Errors in the pressure and VOF fraction ( $\Delta p$ and $\Delta \alpha$ ) induce an error in the bulk density $(\Delta \rho)$, which, given (6b), satisfies the equation

$$
\rho+\Delta \rho=(\alpha+\Delta \alpha) \rho_{u^{\prime}}(p+\Delta p)+(1-\alpha-\Delta \alpha) \rho_{a}(p+\Delta p)
$$

For the model flow and discretization method considered in Section 4.1, we found $\Delta \rho=0$ and $\Delta \alpha=\mathcal{O}(1)$. Then, according to (26), $\Delta p=\mathcal{O}(1)$ as well, which agrees with what we derived in Section 4.1. Instead of the zeroth-order pressure error $\Delta p$ described by (23) and (24), we ideally prefer $\Delta p=0$, which implies according to (26) that

$$
\Delta \rho=\Delta \alpha\left(\rho_{u^{\prime}}(p)-\rho_{a}(p)\right)
$$

One of the fixes considered in the following section is to make the numerical method such that the updates $\Delta \rho$ and $\Delta \alpha$ exactly satisfy (27).

\section{FIXES FOR ERROR IN CELL WITH INTERFACE}

\subsection{Advection of Level-Set Function}

This approach is based on the observation made in Section 4.1 that the update of $\phi_{i}^{n}$ through division of $(\rho \phi)_{i}^{n+1}$ by $\rho_{i}^{n+1}$ leads to an $\mathcal{O}(h)$ accuracy barrier in $\phi_{i}^{n+1}$ because of the intrinsic smearing in the bulk-density representation itself. For the update of the real physical quantities $\rho_{i}$ and $(\rho u)_{i}$ we may stick to the conservative formulation and, hence, to the linearized, two-fluid Osher scheme. Doing so, with the forward-Euler, first-order upwind discretization of the single advection equation

$$
\int_{\Omega_{t}} \frac{\partial \phi}{\partial t} d x+u_{i}\left(\phi_{i+\frac{1}{2}}-\phi_{i-\frac{1}{2}}\right)=0
$$


for the model flow considered-in addition to $\rho_{i}^{n+1}$ and $(\rho u)_{i}^{n+1}$ according to (20)-we get

$$
\phi_{i}^{n+1}=-\frac{h}{2}+\sigma h,
$$

which is exact (because $\phi$ has been defined as the signed-distance function). Because $\Delta \phi_{i}^{n+1}=0$, it also holds that $\Delta \alpha_{i}^{n+1}=0$. Since $\Delta \rho_{i}^{n+1}=0$ as well (Section 4.1), it then follows from (26) that $\Delta p_{i}^{n+1}=0$. In all other cells. the fully conservative scheme (19) is applied, yielding the exact discrete solution. However, at time level $n+2$ the numerical solution is no longer exact: $\rho_{i}^{n+2}=2 \sigma \rho_{u}+(1-2 \sigma) \rho_{u}-\sigma^{2}\left(\rho_{u}-\rho_{u}\right)$, whereas $\left(\rho_{i}^{n+2}\right)_{\text {exact }}=2 \sigma \rho_{u}+(1-2 \sigma) \rho_{a l}$. Hence, $\Delta \rho_{i}^{n+2}=-\sigma^{2}\left(\rho_{u^{\prime \prime}}-\rho_{u}\right)=\mathcal{O}(1)$. With the forward-Euler, first-order upwind discretization of (28), $\Delta \phi_{i}^{n+2}=0$ and, as a consequence, $\Delta \alpha_{i}^{n+2}=0$. With (26) and $\Delta \rho_{i}^{n+2}=\mathcal{O}(1)$, it then follows that $\Delta p_{i}^{n+2}=\mathcal{O}(1)$. So, this partially conservative approach is not a fix. With some tricks one can make the method work. Taking for the left and right cell-face densities to be substituted into the linearized, two-fluid Osher scheme, instead of the bulk densities, the local cell-face densities (pure water or pure air) at $t=t^{n}$, the method works as long as the interface does not cross a cell face during a time step. That is, the method works for $\sigma=1 / m$, with $m$ an integer. Unfortunately, this requirement on $\sigma$ is too restrictive for the method to be of practical use.

\subsection{Advection of Velocity, Pressure and Level-Set Function}

Taking in (25) $q_{i}=\left(u_{i}, p_{i}, \phi_{i}\right)$, with $\left(u_{i-\frac{1}{2}}, p_{i-\frac{1}{2}}\right)=\left(u_{i+\frac{1}{2}}, p_{i+\frac{1}{2}}\right)$, the exact result becomes

$$
\left(\begin{array}{l}
u \\
p \\
\phi
\end{array}\right)_{i}^{n+1}=\left(\begin{array}{l}
u \\
p \\
\phi
\end{array}\right)_{i}^{n}+\left(\begin{array}{c}
0 \\
0 \\
\sigma h
\end{array}\right)=\left(\begin{array}{c}
U \\
P \\
-\frac{h}{2}+\sigma h
\end{array}\right) .
$$

However, in cell $\Omega_{i+1}$ an error arises; $q_{i+1}^{n+1}$ and $q_{i+1}^{n+2}$ are still exact, $q_{i+1}^{n+1}=\left(U, P,-\frac{3}{2} h+\right.$ $\sigma h)$ and $q_{i+2}^{n+1}=\left(U, P,-\frac{5}{2} h+\sigma h\right)$. But for $t=t^{\prime \prime+2}$ we find that $\rho_{i+1}^{n+2}=\rho_{u}+\sigma^{2}\left(\rho_{u}-\rho_{u}\right)$. For $\sigma<1 / 2$, this is wrong; water is erroneously transported from cell $i$ into cell $i+1$. The corresponding error reads $\Delta \rho_{i}^{n+2}=\sigma^{2}\left(\rho_{u}-\rho_{u}\right)=\mathcal{O}(1)$. Meanwhile, for $\sigma<1 / 2$ such that $\phi_{i+\frac{1}{2}}^{n+2}=\phi_{i+\frac{2}{3}}^{n+\frac{2}{3}}$ are both still negative, we correctly find $\alpha_{i+1}^{n+2}=0$. So, with (26) it then follows that $\Delta p_{i+1}^{n+2}=\mathcal{O}(1)$ and therefore this approach is not viable.

\subsection{Advection of Density and Algebraic Update of VOF Fraction}

In Section 4.2 it was shown that if the updates $\Delta \rho$ and $\Delta \alpha$ are such that (27) is satisfied, then $\Delta p=0$. We now will try a possible fix based on (27). Consider the advection equation

$$
\int_{\Omega_{i}} \frac{\partial \rho}{\partial t} d x+u_{i}\left(\rho_{i+\frac{1}{2}}-\rho_{i-\frac{1}{2}}\right)=0
$$

The updates for $\rho_{i}$, rendered by (31), may be directly translated through (27) into updates for $\alpha_{i}$. However, as with the fully nonconservative approach from Section 5.2, in the second time step an $\mathcal{O}(1)$ pressure error arises in neighboring cell $\Omega_{i+1}$, when the fully conservative approach is still applied there. Moreover, even in a better case, the present approach will yield an exact pressure solution at the expense of a diffused density profile. 
In [15], a VOF-fraction formulation is considered which is suitable for the computation of both mixtures of fluids and (immiscible) multifluids and which appears not to suffer from the pressure-oscillation problem. This is probably due to the fact that, as mentioned in Section 3.4 of [15], boundary conditions are imposed at the interface (tracking rather than capturing).

\subsection{Ghost-Fluid Method}

A perfect fix for the pressure-oscillation problem is a simple variant of the ghost-fluid method [5]. In [5], the ghost-fluid method is introduced for the nonhomentropic Euler equations of gas dynamics. For our more compact system of fluid flow equations, we propose a variant, which is comparable to that proposed by Abgrall and Karni [2].

In updating the finite-volume solutions with a single explicit time step, the following is done. For convenience, suppose we have an equidistant, cell-centered finite-volume grid $\Omega_{i}, i=1,2, \ldots, N$, with the cell faces denoted by $\partial \Omega_{i+\frac{1}{2}}, i=0,1, \ldots, N$, with $\partial \Omega_{\frac{1}{2}}$ and $\partial \Omega_{N+\frac{1}{2}}$ the faces at the domain boundaries. Also suppose that at time level $n$ we have a known, unique solution $\left(u_{i}^{n}, p_{i}^{n}, \phi_{i}^{n}\right), i=1,2, \ldots, N$. Then, as first step, at the actual time level $n$, the cells and cell faces are classified into different types. For cells, the following three types are distinguished: (i) pure-water cells, (ii) pure-air cells, and (iii) cells with one (or two) interface(s). To make this classification, we determine $\phi_{i+\frac{1}{2}}^{n}, i=0,1, \ldots, N$. At the interior faces we take

$$
\phi_{i+\frac{1}{2}}^{n}=\frac{1}{2}\left(\phi_{i}^{n}+\phi_{i+1}^{n}\right) . \quad i=1,2, \ldots, N-1 .
$$

At the inflow-boundary face, say $\partial \Omega_{\frac{1}{2}}$, we take

$$
\phi_{\frac{1}{2}}^{n}=\phi_{b}^{n}
$$

with $\phi_{b}^{\prime \prime}$ denoting the boundary condition, and at the outflow boundary, say $\partial \Omega_{N+\frac{1}{2}}$,

$$
\phi_{N+\frac{1}{2}}^{n}=\phi_{N}
$$

Then, cell $\Omega_{i}$ is (i) a pure-water cell if $\phi_{i}^{n}>0, \phi_{i-\frac{1}{2}}^{n}>0$, and $\phi_{i+\frac{1}{2}}^{n}>0$, (ii) a pure-air cell if $\phi_{i}^{n}<0, \phi_{i-\frac{1}{2}}^{n}<0$, and $\phi_{i+\frac{1}{2}}^{n}<0$, or (iii) a cell with one or two interfaces. Cells of the third type are named ghost cells. This classification is also applied to the cell faces; (i) pure-water, (ii) pure-air, and (iii) ghost faces are distinguished. The two faces of a ghost cell are both identified as ghost faces. That is, if $\Omega_{i}$ is a ghost cell, then both $\partial \Omega_{i-\frac{1}{2}}$ and $\partial \Omega_{i+\frac{1}{2}}$ are ghost faces. A cell face not belonging to a ghost cell is-depending on the sign of $\phi$ at that face-either a pure-water or a pure-air face. Across the latter two types of faces, the flux is simply computed with the single-fluid flux formula

$$
F\left(\tilde{q}_{(1}, \tilde{q}_{1}\right)=\left(\begin{array}{c}
\rho_{\frac{1}{2}} u_{\frac{1}{2}} \\
\rho_{\frac{1}{2}} u_{\frac{1}{2}}^{2}+p_{\frac{1}{2}}
\end{array}\right) \text {. with either } \rho_{\frac{1}{2}}=\rho_{u^{\prime}}\left(p_{\frac{1}{2}}\right) \text { or } \rho_{\frac{1}{2}}=\rho_{a}\left(p_{\frac{1}{2}}\right) \text {, }
$$

where the left and right cell-face states are expressed as $\tilde{q} \equiv(u, p)$. As in (14), $u_{\frac{1}{2}}$ and $p_{\frac{1}{2}}$ are given by $(12)$, but now with either $\left(C_{0}, C_{1}\right)=\left(C_{0}, C_{1}\right)_{u^{\prime}}$ (pure water) or $\left(C_{0}, C_{1}\right)=$ 
$\left(C_{0}, C_{1}\right)_{a}$ (pure air). So, across pure-water faces we get $F=F_{u}$ and across pure-air faces $F=F_{a}$. Across the ghost faces two fluxes are computed: a water and an air flux, i.e., both

$$
F_{u^{\prime}}\left(\tilde{q}_{0}, \tilde{q}_{1}\right)=\left(\begin{array}{c}
\rho_{\frac{1}{2}} u_{\frac{1}{2}} \\
\rho_{\frac{1}{2}} u_{\frac{1}{2}}^{2}+p_{\frac{1}{2}}
\end{array}\right), \quad \rho_{\frac{1}{2}}=\rho_{u^{\prime}}\left(p_{\frac{1}{2}}\right)
$$

and

$$
F_{a}\left(\tilde{q}_{0}, \tilde{q}_{1}\right)=\left(\begin{array}{c}
\rho_{\frac{1}{2}} u_{\frac{1}{2}} \\
\rho_{\frac{1}{2}} u_{\frac{1}{2}}^{2}+p_{\frac{1}{2}}
\end{array}\right), \quad \rho_{\frac{1}{2}}=\rho_{a}\left(p_{\frac{1}{2}}\right)
$$

with $u_{\frac{1}{2}}$ and $p_{\frac{1}{2}}$ given by (12) and for (34a), $\left(C_{0}, C_{1}\right)=\left(C_{0}, C_{1}\right)$ and for $(34 \mathrm{~b}),\left(C_{0}, C_{1}\right)=$ $\left(C_{0}, C_{1}\right)_{a}$. Applying the forward-Euler scheme, the subsequent update of finite-volume solutions-expressed in conservative variables, $q=(\rho, \rho u)$-reads

(i) in pure-water cells,

$$
q_{i}^{n+1}=q_{i}^{n}-\frac{\Delta t}{h}\left(\left(F_{u}\right)_{i+\frac{1}{2}}^{n}-\left(F_{u}\right)_{i-\frac{1}{2}}^{n}\right)
$$

(ii) in pure-air cells,

$$
q_{i}^{n+1}=q_{i}^{n}-\frac{\Delta t}{h}\left(\left(F_{a}\right)_{i+\frac{1}{2}}^{n}-\left(F_{a}\right)_{i-\frac{1}{2}}^{n}\right), \text { and }
$$

(iii) in ghost cells,

$$
\begin{aligned}
& \left(q_{w}\right)_{i}^{n+1}=q_{i}^{n}-\frac{\Delta t}{h}\left(\left(F_{u}\right)_{i+\frac{1}{2}}^{n}-\left(F_{u}\right)_{i-\frac{1}{2}}^{n}\right) \text { and } \\
& \left(q_{a}\right)_{i}^{n+1}=q_{i}^{n}-\frac{\Delta t}{h}\left(\left(F_{a}\right)_{i+\frac{1}{2}}^{n}-\left(F_{a}\right)_{i-\frac{1}{2}}^{n}\right) .
\end{aligned}
$$

So, in ghost cells we are left with two possibly different updated solutions: $q_{u}$ and $q_{a}$. Expressed in $\tilde{q}=(u, p)$ variables, these two new ghost solutions will not differ very much for the flows considered here. For the 1D problem introduced in Section 4. 1, both solutions will be identical. (When allowing shock waves, the two solutions in a ghost cell may significantly differ from each other, if the ghost cell also contains a shock wave.) In case a solution ambiguity arises, we proceed as follows. From the updated level-set function (updated separately through an advection equation), the VOF fraction $\alpha_{i}^{n+1}$ in the ghost cell can be computed. Next, the updated solution in the ghost cell is simply made unique with

$$
\tilde{q}_{i}^{n+1}=\alpha_{i}^{n+1}\left(\tilde{q}_{u}\right)_{i}^{n+1}+\left(1-\alpha_{i}^{n+1}\right)\left(\tilde{q}_{u}\right)_{i}^{n+1} .
$$

There are no physical or mathematical arguments for applying this weighting; other choices are possible.

In the computation of the fluxes, we use the same unique values of $u$ and $p$ in each cell, which ensures that the free-surface conditions are satisfied implicitly. In [5], for the nonhomentropic Euler equations of gas dynamics, entropy is extrapolated across the interface. The present homentropic equations require no solution-component extrapolation. 
In the ghost-fluid method the interface is no longer captured at the lowest discrete level, that of the cell face, but at the next higher level, the cell. Because fluxes are always of the single-fluid type in the ghost-fluid method, explicit calculation of $u_{\frac{1}{2}}$ and $p_{\frac{1}{2}}$ can be easily done by using, e.g., the full, nonlinear Osher scheme instead of a linearized scheme.

\section{NUMERICAL RESULTS}

\subsection{Water Front at Constant Speed and Pressure}

The first test case to be considered is the ID tube flow from Section 4.1. Numerical values to be used are $\left(x_{\mathrm{fs}}\right)_{t=0}=0.5$ (initial interface halfway tube) and $\left(x_{\mathrm{fs}}\right)_{t=0}=0$ (initial interface at inlet boundary), $U=1, P=1, \rho_{u}(P)=1, \rho_{u}(P)=0.001$ (other values are considered as well), $\gamma_{u}=7, \gamma_{a}=7 / 5, B_{u}=3000$, and $B_{a}=0$. According to the speedof-sound relations

$$
c_{u}^{2}=\gamma_{u} \frac{\left(1+B_{u}\right) p}{\rho_{u}}, \quad c_{u}^{2}=\gamma_{a} \frac{p}{\rho_{a}} .
$$

these values imply $c_{u}(P) \approx \sqrt{15} c_{a}(P)$, which agrees fairly well with standard sea-level conditions. As in Section 4 , the grids to be used are equidistant. The boundary conditions to be imposed are $u(x=0, t)=U, \phi(x=0, t)=U t$, and $p(x=1, t)=P$. The space discretization is first-order accurate, as in Section 4.1. Time integration is done with the forward-Euler scheme, with the time step constant and sufficiently small to guarantee stability:

$$
\Delta t=\sigma \frac{h}{U+c_{w}(P)} . \quad \sigma<1 .
$$

6.1.1. Fully conservative approach. This is the approach without any fixes for solution errors near the interface. For the above numerical values, the computation breaks down. The stumbling block is the large density ratio. In Fig. 4, pressure errors are depicted for computations with three still rather small density ratios, $\rho_{u^{\prime}} / \rho_{a}=2,4$, and 8 , after 10 , 20, and 40 time steps. The time step on the coarsest grid is twice as large as that on the middle grid and four times larger than that on the finest grid. No results were obtained for $\rho_{u} / \rho_{a}=8$ after 40 time steps. The pressure error appears to be about linearly proportional to the number of time steps taken. In agreement with the theoretical findings, it also increases with the density ratio $\rho_{u^{\prime}} / \rho_{a}$. The latter increase is clearly nonlinear. With the conservative approach, results for $\rho_{u} / \rho_{a}=1000$ are still far out of reach. The deceptive performance of the conservative approach was expected given the analytical results of Section 4.1. (The behavior is typical for most conservative formulations but not intrinsic to all [3].)

6.1.2. Advection of level-set function. Here the fix proposed in Section 5.1 is numerically investigated. It is applied not only in the cell in which the interface actually is, but also in its left and right neighbor cells. The fix clearly gives an improvement as compared to the fully conservative approach but is not adequate. For $\left(x_{\mathrm{fs}}\right)_{t=0}=0$ and $\rho_{u^{\prime}} / \rho_{a}=10$, in each of the three graphs in Fig. 5 we present the computed bulk-density profiles at $t=0.0$, $0.1,0.2, \ldots, 1.0$. The results are perfect as far as the capturing of the interface is concerned; capturing over a single mesh width $h$ only (thanks to the level-set approach). However, they 
After 10 time steps

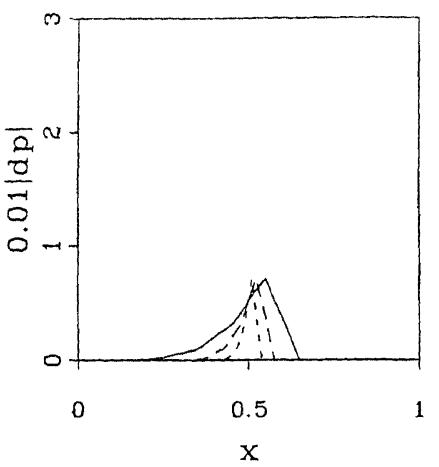

$\mathrm{x}$

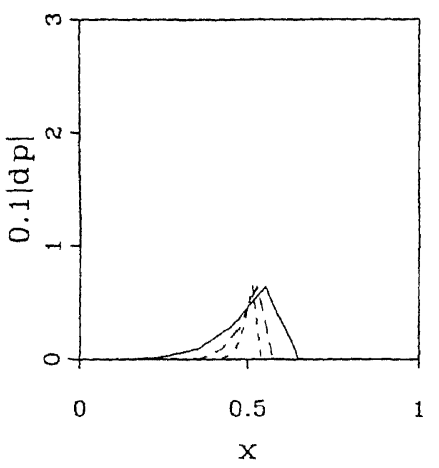

$x$

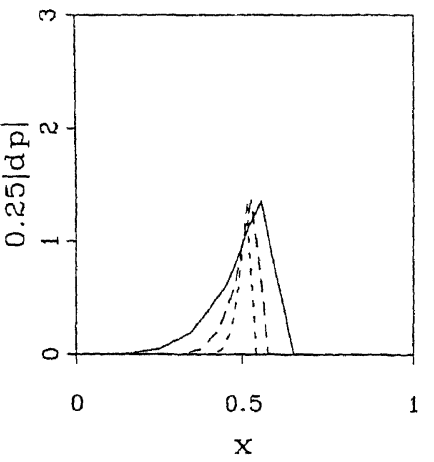

After 20 time steps

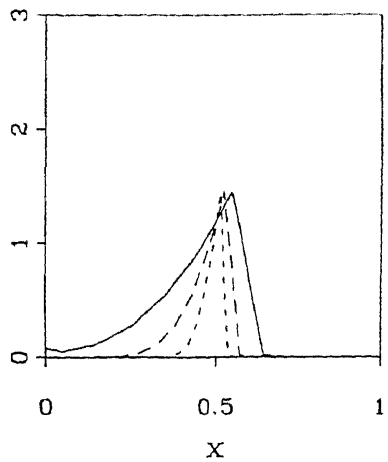

a. $\frac{p_{w}}{p_{a}}=2$

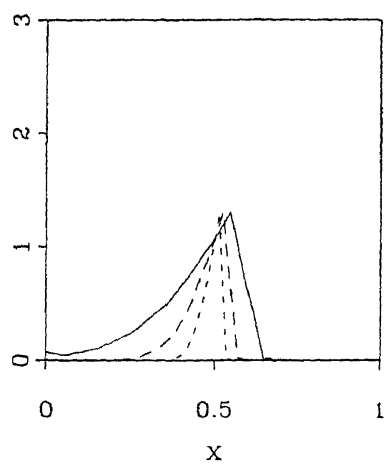

b. $\frac{\rho_{w}}{\rho_{a}}=4$

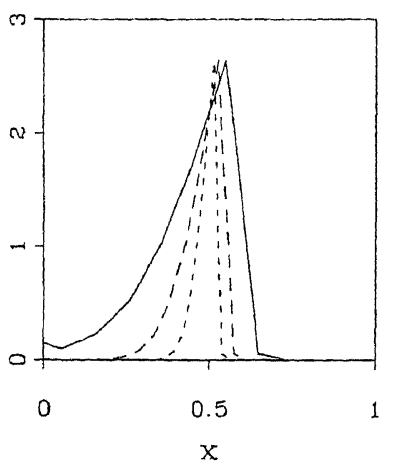

c. $\frac{\rho_{w}}{\rho_{a}}=8$
After 40 time steps
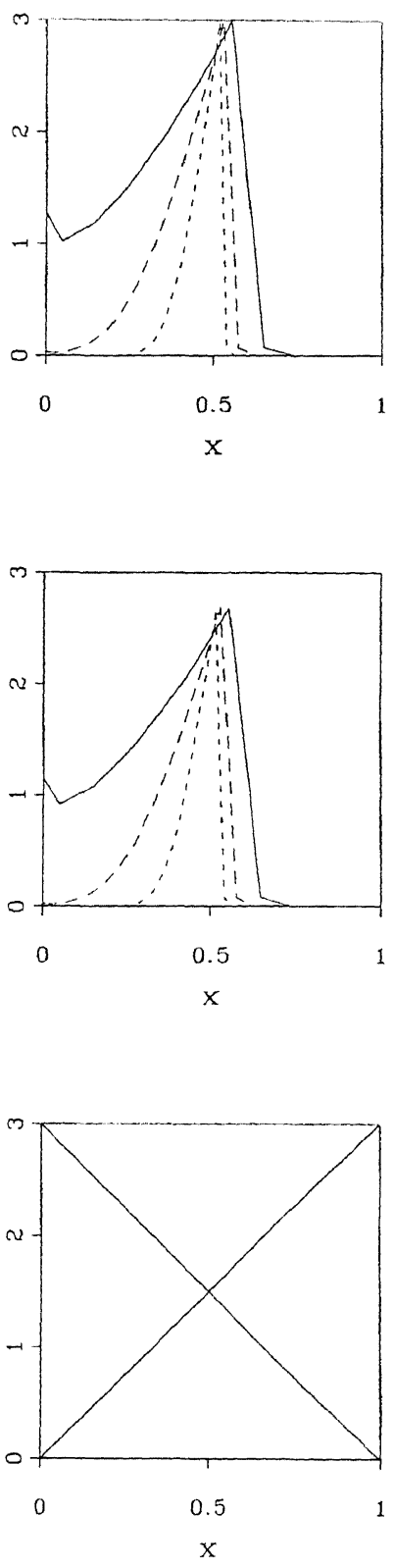

FIG.4. Pressure-error distributions by the fully conservative approach (solid lines, $h=1 / 10$ ); coarsely dashed lines, $h=1 / 20$; finely dashed lines, $h=1 / 40$ ).

are cursed with a pressure error, which for $\rho_{w} / \rho_{a}=10$ is still negligibly small, but which, as in the previous section, grows nonlinearly with increasing density ratio $\rho_{u} / \rho_{u}$. Results similar to those in Fig. 5 cannot be obtained for $\rho_{w} / \rho_{u}=1000$, not even for $\rho_{w} / \rho_{u}=100$. From Fig. 6 it appears that the pressure error grows exponentially with $\rho_{w} / \rho_{u}$. 


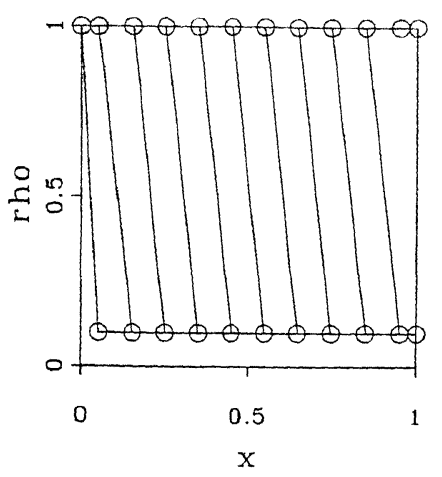

a. $h=\frac{1}{10}$

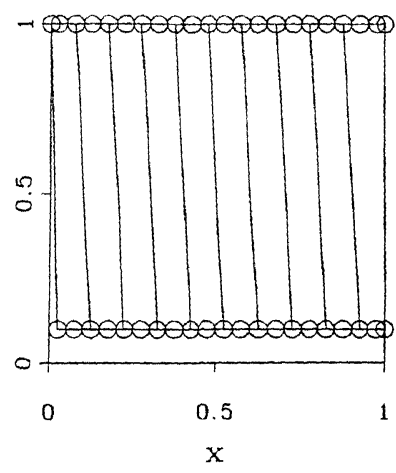

b. $h=\frac{1}{20}$

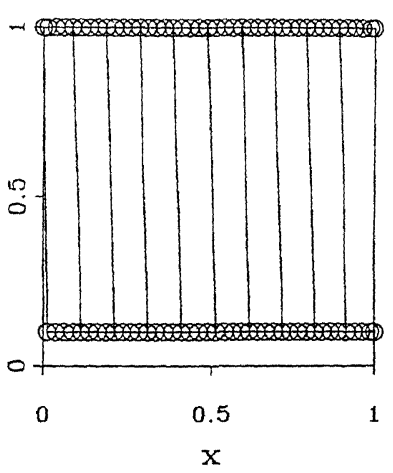

c. $h=\frac{1}{40}$

FIG. 5. Bulk-density profiles at $t=0.0,0.1,0.2 \ldots, 1.0$, fix with advection of level-set function, $\rho_{u} / \rho_{a}=10$.

6.1.3. Advection of velocity, pressure, and level-set function. Here, the fix proposed in Section 5.2 is tested. The advection of $u, p$, and $\phi$ is applied to the cell with the interface as well as to its left and right neighboring cells. The fix is an improvement compared to that with advection of $\phi$ only, but it does not work satisfactorily either. Capturing is again perfect, but the method also breaks down for increasing density ratio $\rho_{u^{\prime}} / \rho_{a}$, for $\rho_{w^{\prime}} / \rho_{a}=100$ after $t=0.7$, and for $\rho_{u} / \rho_{a}=1000$ after $t=0.4$ (Fig. 7).

6.1.4. Ghost-fluid method. The fix proposed in Section 5.3 is not tested; its expected smearing of the density excludes it as an interesting option here. However, the ghost-fluid method described in Section 5.4 is interesting. It works (Fig. 8); it gives perfectly sharp interfaces and does not break down with increasing density ratio. As opposed to the previous methods it works for standard water-air conditions, $\rho_{u} / \rho_{a}=\mathcal{O}\left(10^{3}\right)$. For the problem at hand, it even works for arbitrarily large density ratios (Fig. 9). Reinitialization of the level-set function is not necessary for the running water front.

\subsection{Oscillating Water Column}

Although the previous constant-speed-and-pressure test case is not trivial from a numerical point of view, from a physical perspective it is. For the second test case, we consider

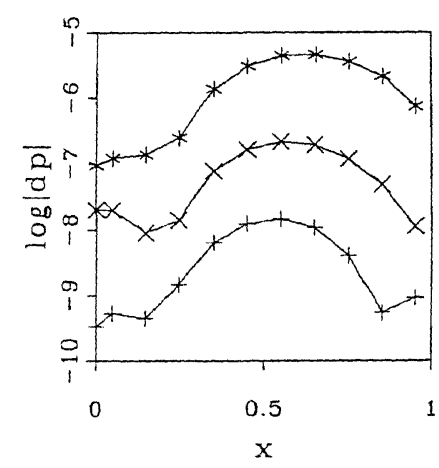

FIG. 6. Pressure-error distributions at $t=0.1$ for $\rho_{u} / \rho_{a}=70(+), 75(\times)$, and $80(*)$, fix with advection of level-set function, $h=1 / 40$. 


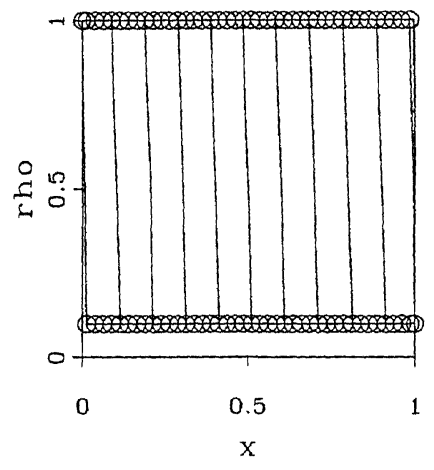

a. $\frac{\rho_{w}}{\rho_{a}}=10$

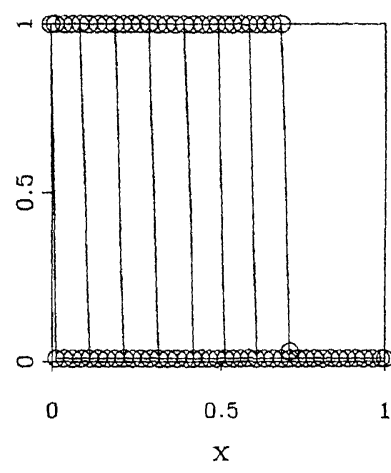

b. $\frac{\rho_{w}}{\rho_{a}}=100$

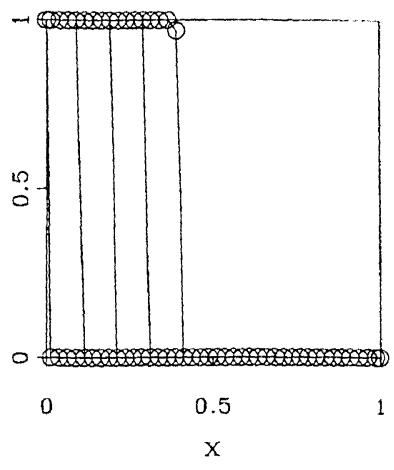

c. $\frac{\rho_{w}}{\rho_{a}}=1000$

FIG. 7. Bulk-density profiles at $t=0.0,0.1,0.2, \ldots, 1.0$, fix with advection of velocity, pressure, and level-set function, $h=1 / 40$.

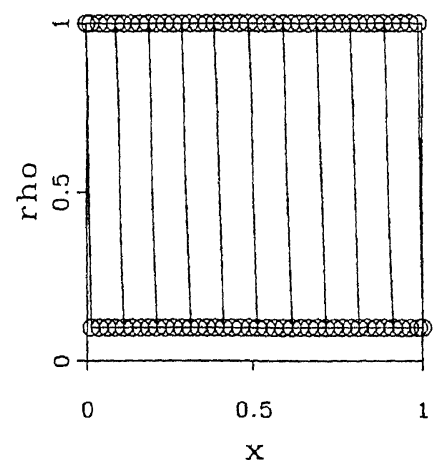

a. $\frac{p_{w}}{p_{a}}=10$

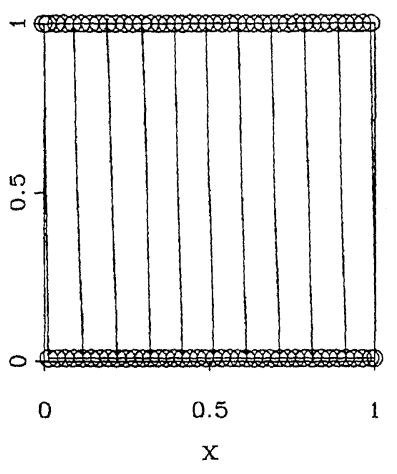

b. $\frac{\rho_{w}}{\rho_{a}}=100$

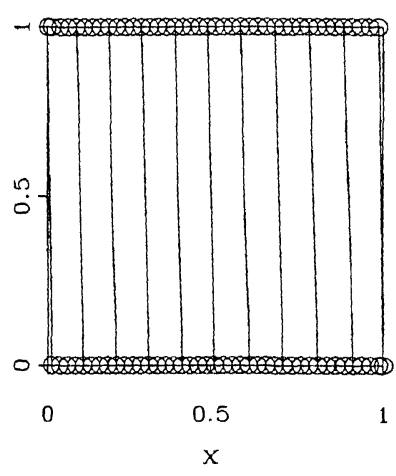

c. $\frac{\rho_{w}}{\rho_{a}}=1000$

FIG. 8. Bulk-density profiles at $t=0.0,0.1,0.2, \ldots, 1.0$, ghost-fluid method, $h=1 / 40$.

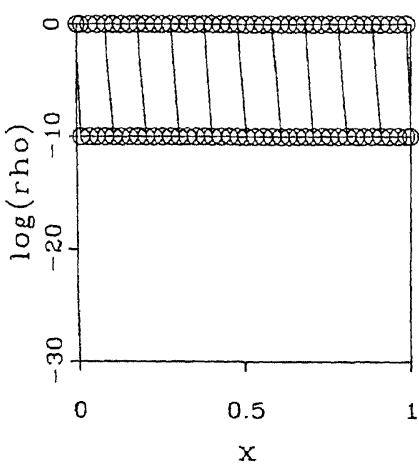

a. $\frac{\rho_{w}}{\rho_{a}}=10^{10}$

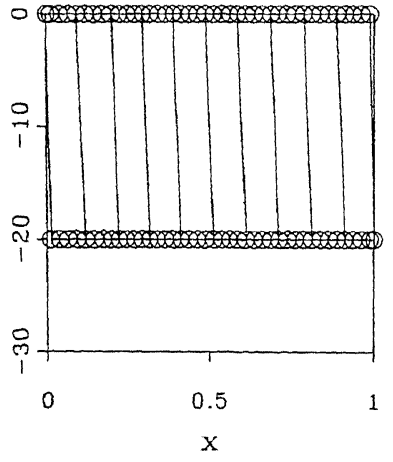

b. $\frac{\rho_{w}}{\rho_{a}}=10^{20}$

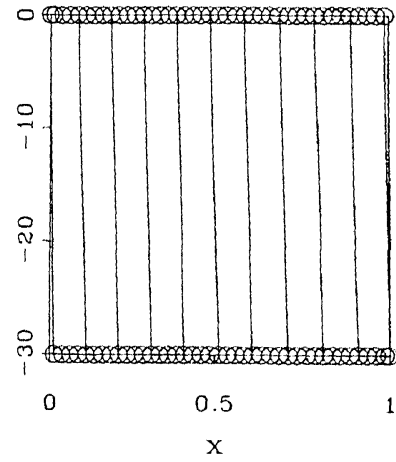

c. $\frac{\rho_{w}}{\rho_{a}}=10^{30}$

FIG. 9. Bulk-density profiles at $t=0.0,0.1,0.2, \ldots, 1.0$, ghost-fluid method, extremely high density ratios. $h=1 / 40$. 


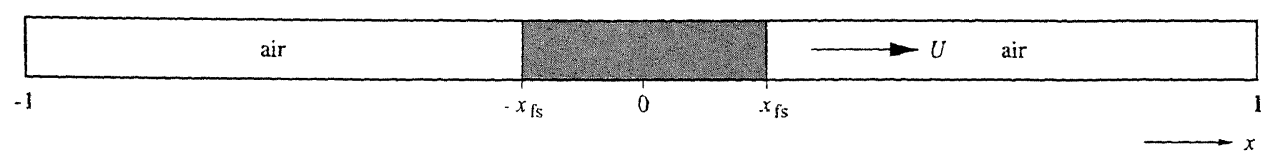

FIG. 10. Initial condition: shut off tube with column of water (grey) in between two columns of air, all three columns flowing to the right at constant speed $U$ and pressure $P$.

a closed ID tube (i.e., with impermeable boundaries at the left and right), with the initial solution as sketched in Fig. 10. Starting from $t=0$, the air at the right is compressed by the water and the air at the left expands. Hence, a pressure difference is built up across the column of water, with a consequent deceleration of the latter's flow to the right, followed by stagnation, and then an acceleration and flow to the left. This leads to a reverse pressure gradient across the water, which redirects the flow from left to right again, and so on. The water column starts to oscillate.

We present numerical results obtained through the ghost-fluid method. As for the previous test case, we take $\gamma_{u^{\prime}}=7, \gamma_{a}=7 / 5, B_{u^{\prime}}=3000, B_{a}=0, \rho_{u^{\prime}}(P)=1$, and $\rho_{a}(P)=0.001$. Further, we take $U=1, P=1$, and $x_{\mathrm{fs}}=0.1$.

6.2.1. Pressure behavior. An equidistant grid with $h=1 / 40$ is applied. The space discretization is again first-order accurate and time integration is done again with the forwardEuler scheme. The level-set function is taken as the signed-distance function. For this test case, as opposed to the foregoing, the level-set function is reinitialized. (The reinitialization is done after each time step.) Fig. 11 shows the time evolution of the pressure coefficients

$$
\mathcal{P}(x=-1, t) \equiv \frac{p(x=-1, t)-P}{P}, \quad \mathcal{P}(x=1, t) \equiv \frac{p(x=1, t)-P}{P} .
$$

The ghost-fluid method appears to work fine.

6.2.2. Conservation errors. In the ghost cells, the conservation laws are applied to ghost (i.e., virtual) amounts of water and air, not to the real physical amounts. Therefore, conservation of the real amounts of mass and momentum in these cells is not guaranteed.

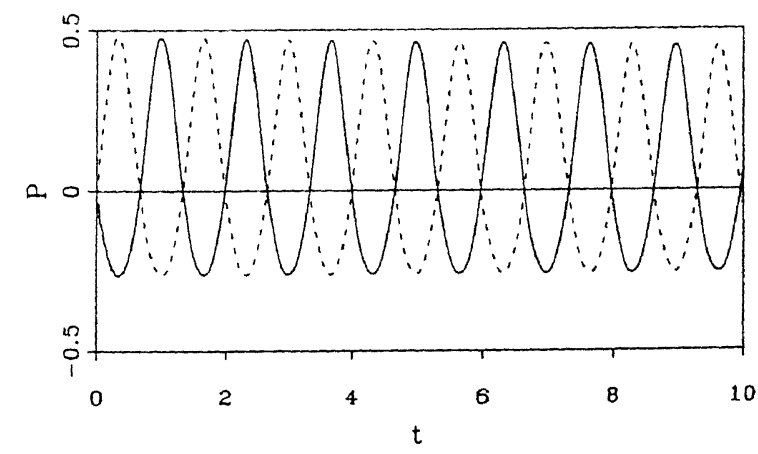

FIG. 11. Time evolution of pressure coefficients at left and right boundaries (solid line, left boundary; dashed line, right boundary), $h=1 / 40$. 


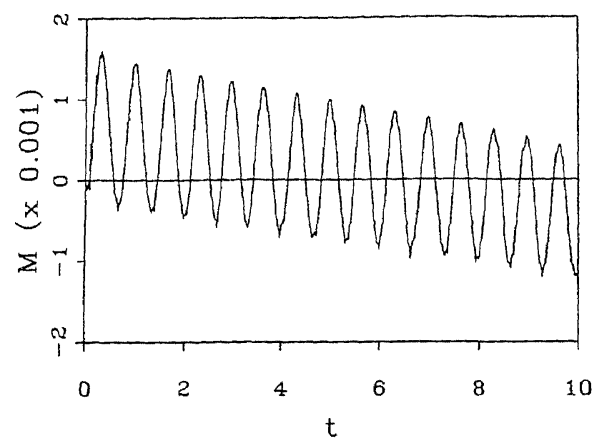

a. $h=\frac{1}{40}$

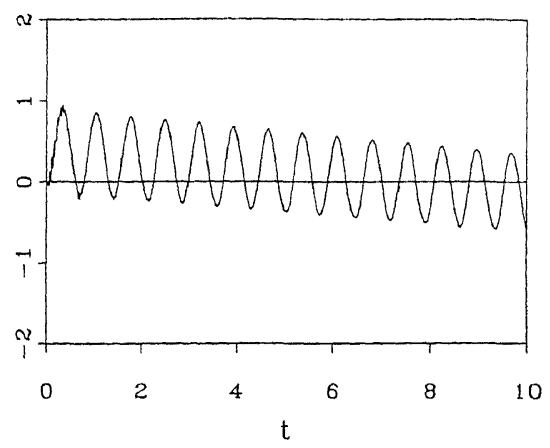

b. $h=\frac{1}{80}$

FIG. 12. Time evolution of relative error in total mass of air in shut off tube.

In Fig. 12a we give the time evolution of the relative mass error

$$
\mathcal{M}(t) \equiv \frac{m_{a}(t)-m_{a}(0)}{m_{a}(0)},
$$

where $m_{a l}(t)$ is the total mass of air in the tube at time $t$. The air-mass error appears to be composed of two components: one oscillating and the other behaving linearly in time. The total mass of air is slowly decreasing; air is being converted into water. Fortunately, the orders of both the oscillatory and the linear error component are close to the computational method's order of accuracy, which is first order. To show the latter, in Fig. 12b the time evolution of the relative air-mass error is given for a grid and time step twice as fine as those in Fig. 12a. (The orders of accuracy of the oscillatory and linear error component in going from $h=1 / 40$ to $h=1 / 80$ appear to be 0.78 and 0.90 , respectively.)

\section{EXTENSION TO MULTIPLE DIMENSIONS}

In this section, the extension to multi-D of the best performing method, the ghost-fluid method, is outlined. For convenience, we consider the extension to 2D only. The subsequent extension to 3D is rather straightforward. We consider quadrilaterals as finite volumes in 2D.

\subsection{Interface Location and VOF Fraction}

As in 1D, to identify cells intersected by the two-fluid interface (ghost cells), we first interpolate the cell-center values of the level-set function $\phi$ to the cell vertices. For sufficiently smooth grids, this interpolation may be simply done as

$$
\phi_{i+\frac{1}{2}, j+\frac{1}{2}}=\frac{1}{4}\left(\phi_{i, j}+\phi_{i+1 . j}+\phi_{i, j+1}+\phi_{i+1, j+1}\right) .
$$

(If desired, it may be done more accurately by grid-dependent bilinear interpolation.) Knowing the values of $\phi$ in the cell centers and cell vertices, we can determine for each cell the VOF fraction $\alpha$ in the following way. If for a cell, $\phi$ has the same sign in its center and four vertices (e.g., a positive sign, Fig. 13a), that cell is fully filled with one of the two 


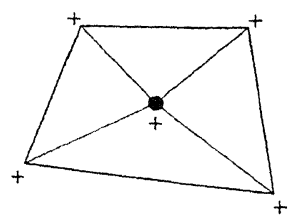

a. All four positive

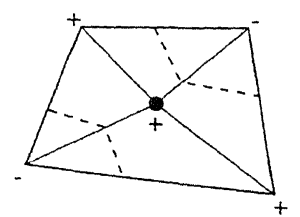

d. Two diagonally opposite negative

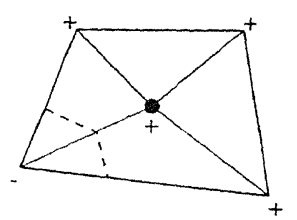

b. One negative

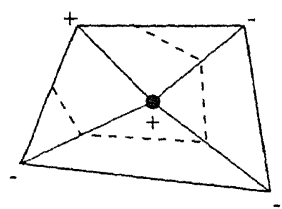

e. Three negative

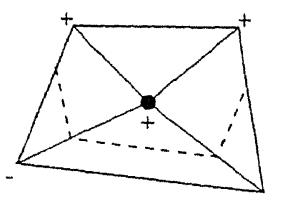

c. Two neighboring negative

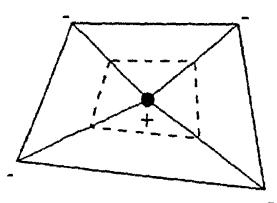

f. All four negative

FIG. 13. Six possible combinations of signs of $\phi$ at the four vertices of a quadrilateral finite volume, assuming $\phi$ to be positive in the finite volume's center (dashed lines indicate the two-fluid interface).

fluids ( $\alpha=1$ or $\alpha=0$ ). If $\phi$ does not have the same sign in its center and four vertices, we assume linear distributions of $\phi$ along the cell faces connecting two vertices with different sign of $\phi$, as well as along the line segments connecting the cell center and a cell vertex with different sign of $\phi$. Given these linear distributions, the locations of the interface along these cell faces and line segments can be determined. Next, assuming a piecewise linear shape of the interface in between the latter locations (Figs. 13b-13f), the VOF fractions can be calculated.

\subsection{Flux Computation}

In all cells intersected by an interface (ghost cells), as in ID, we compute both a ghostwater and a ghost-air flux across all four faces. Across all other cell faces, depending on the sign of $\phi$, either pure-water or pure-air fluxes are computed. All flux computations can be done in a standard, locally ID manner, i.e., by considering the flux at each cell face as the solution of a Riemann problem normal to that cell face. An ambiguity in the ghost-fluid solution may be removed through a formula analogous to (38).

\section{CONCLUSIONS}

To accurately compute compressible, immiscible two-fluid flows with very large density differences (such as water-air flows), we have proposed a method that uses a level-set technique to distinguish between the two fluids. The level-set equation has been incorporated consistently into the system of hyperbolic conservation laws. The resulting equations have been discretized through a finite-volume method. To compute the fluxes across the finitevolume faces (the level-set flux being one of the flux-vector components), we have proposed a linearized, two-fluid Osher scheme. The scheme allows a physically correct capturing of the interface across a single cell face, as well as a neat boundary-condition treatment (for example, no sticking of interfaces to solid walls). The novel scheme combines good physical properties with great simplicity and efficiency.

To avoid large solution errors near interfaces, which is a problem for many conservative capturing methods, four fixes have been proposed, three consisting of some locally 
advective solution update and the fourth a ghost-fluid fix. For density ratios of order 1000 (typical water-air ratio) the advective fixes fail in the analyses as well as in the numerical experiments. In contrast, the simple ghost-fluid technique does work. Even the computation of fronts running into a vacuum $\left(\rho_{w} / \rho_{a}=\infty\right)$ is expected to be possible with the ghost-fluid method. Extension of the method to higher order accuracy is straightforward through the use of, e.g., a MUSCL approach and a multistage time integrator. (For flow problems such as the running water front considered in this paper, higher order accuracy is not necessary; already the first-order-accurate discretization method-thanks to the level-set approach-captures the interface over the distance of a single mesh width only.) Concerning the extension to higher dimensions of the ghost-fluid method, no principal difficulties exist.

\section{REFERENCES}

1. R. Abgrall, How to prevent pressure oscillations in multicomponent How calculations: A quasi conservative approach, J. Comput. Phys. 125, 150 (1996).

2. R. Abgrall and S. Karni, Computation of compressible multifluids, J. Comput. Phys. 169, 594 (20)()1).

3. E. H. van Brummelen and B. Koren, A pressure-invariant conservative Godunov-type method for barotropic two-fluid flows, submitted for publication.

4. C. H. Cooke and T.-J. Chen, Continuous front tracking with subcell resolution, J. Sci. Comput. 6, 269 (1991).

5. R. P. Fedkiw, T. Aslam, B. Merriman, and S. Osher, A non-oscillatory Eulerian approach to interfaces in multimaterial flows (the ghost fluid method), J. Comput. Phys. 152. 457 (1999)).

6. J. Flores and M. Holt, Glimm's method applied to underwater explosions, J. Comput. Phys. 44, 377 (1981).

7. D. R. van den Heul, C. Vuik, and P. Wesseling, A staggered scheme for hyperbolic conservation laws applied to unsteady sheet cavitation, Comput. Visualiz. Sci. 2. 63(1999).

8. C. W. Hirt and B. D. Nichols, Volume of fluid (VOF) method for the dynamics of free boundaries, $J$. Comput. Phys. 39, 201 (1981).

9. P. Jenny, B. Müller, and H. Thomann, Correction of conservative Euler solvers for gas mixtures, $J$. Comput. Phys. 132, 91 ( 1997 ).

10. S. Karni, Multicomponent flow calculations by a consistent primitive algorithm, J. Comput. Phys. 112. 31 (1994).

11. S. Karni, Hybrid multifluid algorithms, SIAM J. Sci. Comput. 17, 1019 (1996).

12. B. Koren and A. C. J. Venis, A fed back level-set method for moving material-void interfaces, J. Comput. Appl. Math. 101, 131 (1999).

13. S. Osher and F. Solomon, Upwind difference schemes for hyperbolic systems of conservation laws, Math. Comput. 38, 339 (1982).

14. R. Saurel and R. Abgrall, A simple method for compressible multifluid flows, SIAM J. Sci. Complut. 21,1115 (1999).

15. R. Saurel and R. Abgrall, A multiphase Godunov method for compressible multifiuid and multiphase flows, J. Comput. Phys. 150, 425 (1999).

16. J. A. Sethian, Level Set Methods: Evolving Interfaces in Geometry, Fluid Mechanic:s, Computer Vision, and Materials Science (Cambridge Univ. Press, Cambridge, UK, 1996).

17. M. Sussman, P. Smereka, and S. Osher, A level set approach for computing solutions to incompressible two-phase flow, J. Comput. Phys. 114. 146 (1994). 